$\mathrm{ROC}$ curve of $\mathrm{sCr}$ for predicting terlipressin non-response

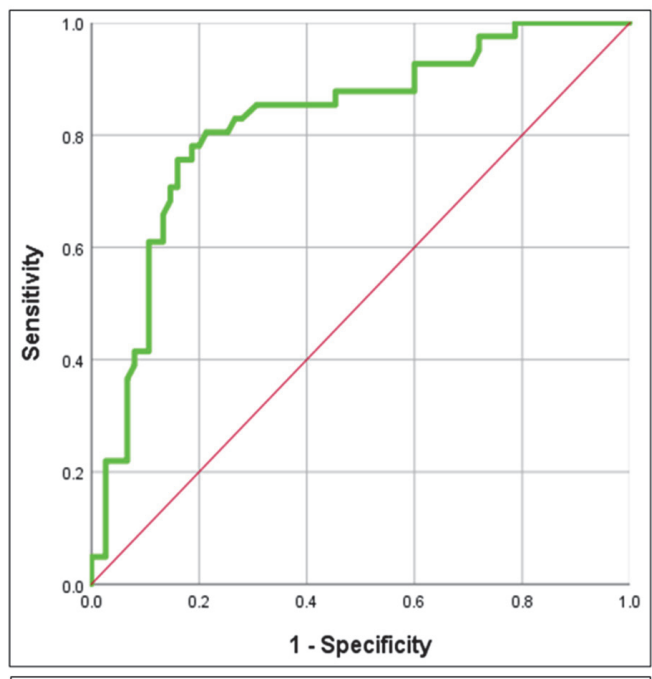

Baseline $\mathrm{sCr} \geq 3.02 \mathrm{mg} / \mathrm{dl}$ had a sensitivity of $75.6 \%$ and specificity of $84 \%$ for predicting terlipressin non-response with a AUROC of 82.2 $(95 \% \mathrm{CI}, 74.1-90.4 ; \mathrm{p}<0.001)$.
ROC curve of MELD score for predicting 90-day mortality

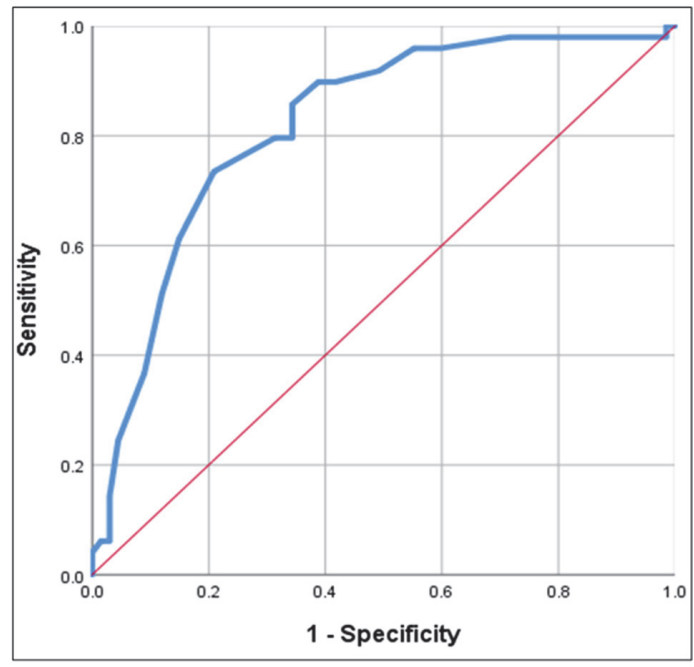

MELD $\geq 30.5$ had a sensitivity of $85.7 \%$ and specificity of $65.7 \%$ for predicting 90-day mortality with AUROC of 81.9 (95\%CI, 74.1-89.7; $\mathrm{p}<0.001)$.

Abstract IDDF2020-ABS-0192 Figure 1 ROC curve of serum creatinine (sCr) and MELD score for predicting terlipressin non-response and 90-day mortality, respectively

MELD score [HR-1.12 (1.06-1.18);p<0.001] predicted mortality at day-90 (figure 1).

Conclusions Terlipressin infusion is safe and effective in ACLF patients. Non-response to terlipressin is dependent on baseline $\mathrm{sCr}$ and ACLF grade. Terlipressin non-response and MELD scores predict mortality in ACLF patients with HRS-AKI. (CTRI/2019/10/021737).

\section{IDDF2020-ABS-0197 STUDY ON CORRELATION BETWEEN SERUM FERRITIN LEVELS AND LIVER STIFFNESS ASSESSED BY FIBROSCAN IN PATIENTS WITH CHRONIC HEPATITIS C}

${ }^{1} \mathrm{Ha}$ Phuong Linh*, ${ }^{2}$ Le Thi Thu Hien, ${ }^{1}$ Dinh Cong Dang, ${ }^{1}$ Hoang Van Tiep. 'Department of Internal Medicine, 103 Cam Khe Clinic, Vietnam; ${ }^{2}$ Department of Internal Medicine, Thai Nguyen University of Medicine and Pharmacy, Vietnam

\subsection{6/gutjnl-2020-IDDF.166}

Background Chronic hepatitis $\mathrm{C}$ is a major infectious disease which mainly causes of morbidity worldwide in patients with liver disease and liver transplantation. Raised ferritin levels play an important role in intervening the process which is associated with hepatic injury. Screening with non-invasive strategies can detect the disease at the early stage, and intervention could be initiated.

Aims To determine the correlation between serum ferritin levels and liver stiffness values in patients of chronic hepatitis C. Methods A cross-sectional study was conducted at 103 Cam Khe Clinic from May 2019 to April 2020. 93 patients with chronic hepatitis $\mathrm{C}$ fulfilling inclusion criteria were included in this study. Liver fibrosis stages were appreciated using transient hepatic elastography by Fibroscan, the activities of serum liver function biomarker enzymes and serum ferritin levels were determined by the automated analyser.

Results The average age of patients was 48 years, with men accounted for $78 \%$ of the total. The mean serum ferritin value was $148.19 \mathrm{ng} / \mathrm{ml}$, liver stiffness measurements range from 12.5 to $75.5 \mathrm{kPa}$, with a median value of $17.39 \pm$ $15.98 \mathrm{kPa}$. Significantly elevated levels of serum ferritin $(\mathrm{p}<$ 0.001), were detected in patients with severe fibrosis compared to mild fibrosis. The concentration of serum ferritin was increased with the evolution of fibrosis in all stages from F0 to $F 4$, and this increase was significant $(p<0.01)$ in cirrhotic patients (F4). There was a positive correlation between serum level of ferritin and the progression of fibrosis (0.979391) ( $\mathrm{r}=0.976)$.

Conclusions There is a significant correlation between serum ferritin and liver stiffness. Serum ferritin concentration may be used as liver fibrosis biomarkers.

\section{IDDF2020-ABS-0198 STUDY ON FIBROSIS CHANGE WITH TRANSIENT ELASTOGRAPHY IN CHRONIC HEPATITIS B VIRUS TREATMENT WITH TENOFOVIR}

${ }^{1}$ Ha Phuong Linh*, ${ }^{2}$ Le Thi Thu Hien, ${ }^{1}$ Hoang Van Tiep, ${ }^{2}$ Dinh Cong Dang, ${ }^{2}$ Nguyen Khac Hung Manh. 'Department of Internal Medicine, 103 Cam Khe Clinic, Vietnam: ${ }^{2}$ Department of Internal Medicine, Thai Nguyen University of Medicine and Pharmacy, Vietnam

\subsection{6/gutjpl-2020-IDDF.167}

Background Tenofovir disoproxil fumarate (TDF) is one of the first optimal choices to be used in the treatment of chronic hepatitis B. FibroScan is non-invasive methods to assess liver fibrosis.

Aims To evaluate the therapeutic effect of TDF on fibrosis via FibroScan after treatment.

Methods This study was conducted in 63 chronic hepatitis B patients who had the indication of antiviral therapy at 103 Cam Khe Clinic from March 2019 to March 2020. All patients with chronic hepatitis B treated with TDF for 6 months. Liver fibrosis stages were appreciated using transient hepatic elastography by Fibroscan before and after 6 months of treatment. 
Results The average age of patients was 46 years, with men accounted for $69 \%$ of the total. After treatment, normalization of ALT $71.26 \%$, the viral response of $90.23 \%$, HBV DNA below the detection level was $66.3 \%$. Liver fibrosis evaluated by FibroScan before and after 6 months treatment were 7.15 $\pm 1.56 \mathrm{kPa}$, and $3.58 \pm 1.19 \mathrm{kPa}$ evaluated by FibroScan. Conclusions TDF was effective for patients after treatment on liver fibrosis assessed by FibroScan in chronic hepatitis B patients.

\section{IDDF2020-ABS-0199 HISTOPATHOLOGICAL AND IMMUNOHISTOCHEMICAL CHARACTERIS- TICS OF HEPATOCELLULAR CARCINOMA WITH PORTAL VEIN THROMBOSIS}

${ }^{1}$ Le Thi Thu Hien*, ${ }^{2}$ Le Quoc Tuan, ${ }^{3}$ Nguyen Quang Duat. 'Department of Internal Medicine, Thai Nguyen University of Medicine and Pharmacy, Vietnam; ${ }^{2}$ Department of Internal Medicine, Thanh Ba Hospital, Vietnam; ${ }^{3}$ Department of Internal Medicine, 103 Military Hospital, Vietnam

\subsection{6/gutjpl-2020-IDDF.168}

Background Detection of portal vein thrombosis (PVT), especially benign or neoplastic PVT in new diagnosed hepatocellular carcinoma (HCC) has key meaning for prognosis as well as making the choice of treatment methods.

Objectives To analysis of histopathological and immunohistochemical characteristics of hepatocellular carcinoma with portal vein thrombosis

(1)To the analysis of histopathological and immunohistochemical characteristics of hepatocellular carcinoma with portal vein thrombosis

(2)To analyse the histopathological and immunohistochemical characteristics of hepatocellular carcinoma with portal vein thrombosis

Methods We conducted a prospective study of 50 patients with HCC have PVT at Clinic 103 Cam Khe from June 2017 to August 2019. The PVT specimens were collected by biopsy through the skin according to ultrasound guidance. Immunohistochemistry test: the dyeing was performed by BondMax automatic dyeing machine (Leica Biosystems - Australia).

Results The location of HCC tumors associated with the location of PVT $(p<0.01)$. Thromboses in the trunk of the portal vein of $56 \%$, thromboses in the branches of the portal vein of $44 \%$. The degree of thrombosis significantly related to the size of HCC tumor $(\mathrm{p}<0.01)$. All of the PVT in patients with HCC were malignant thromboses with moderate differentiation of $58 \%$, poor differentiation of $36 \%$, high differentiation of $6 \%$. Neoangiogenesis in the thromboses by using immunohistochemistry: low level of 6\%, moderate level of $46 \%$ and a high level of $48 \%$. The neoangiogenesis in the thromboses significantly related to cancer cell differentiation $(\mathrm{p}<0.01)$.

Conclusions The more angiogenesis was, the lower the grate of cell differentiation.

\section{IDDF2020-ABS-0210 STATIN USE ASSOCIATED WITH REDUCED RISK OF ALL-CAUSE MORTALITY IN HEPATOCELLULAR CARCINOMA PATIENTS FOLLOWING LIVER RESECTION: A SYSTEMATIC REVIEW AND META- ANALYSIS}

Philip Chun Yeung*, Siu Tim Cheung, Kelvin Kwok-Chai Ng, Paul Bo-San Lai, Charing Ching-Ning Chong. The Chinese University of Hong Kong, Hong Kong

\subsection{6/gutjnl-2020-IDDF.169}

Background The benefit of statins in overall survival of hepatocellular carcinoma (HCC) patients after curative liver resection has been controversial. Some retrospective studies identified perioperative use of statins was associated with significantly reduction in all-cause mortality in HCC patients after liver resection, among patients with or without chronic hepatitis viral infection in multivariate survival analysis. However, there are also several studies conducted in Japan and United States against the claim. To evaluate the effect of statins on the risk of all-cause mortality in HCC patients after curative liver resections, we performed a systematic review and meta-analysis on this topic.

Methods A systematic search of Medline, Embase, Cochrane Library and Web of Science was conducted through August 2020. Studies were included if they evaluated perioperative exposure to statins, reported the all-cause mortality of HCC patients after curative resection and reported adjusted hazard ratios (HR) of multivariable analysis by Cox proportional hazards model. Summary HR estimates with 95\% confidence intervals (CI) were calculated using the random-effects model.

Results The analysis included 4 studies reporting the all-cause mortality of 3,762 HCC patients after liver resection, in which 384 of them received perioperative statins treatments. A meta-analysis of the studies showed a significant (48\%) reduction in the risk of all-cause mortality among HCC patients who had perioperative statins use (adjusted HR, 0.52; 95\% CI, 0.34-0.82), with moderate heterogeneity among studies (Cochran's $\mathrm{Q}$ test, $\mathrm{P}=0.12, \mathrm{I}^{2}=49 \%$ ) (figure 1) with no publication bias. patients after liver resection

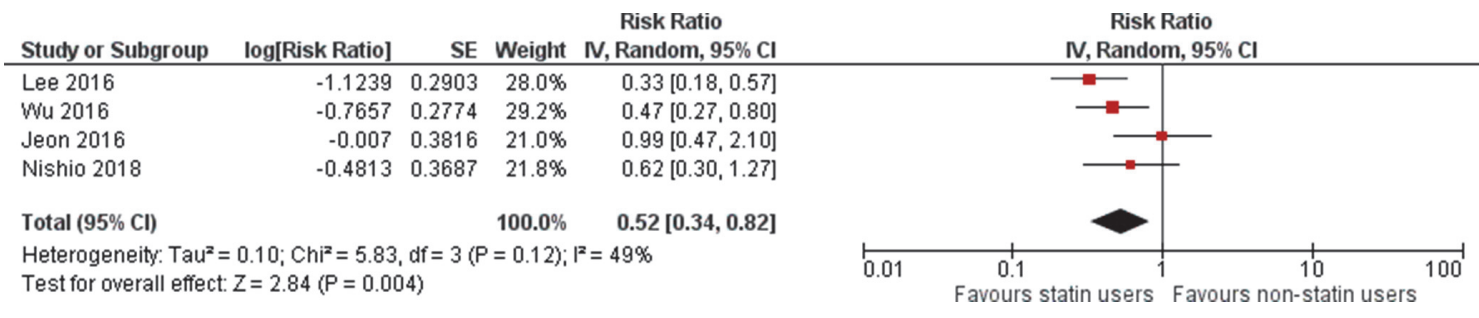

Abstract IDDF2020-ABS-0210 Figure 1 Forest plot for the meta-analysis of the association between statin use and overall survival in HCC 